\title{
Honeywell
}

\section{Fabrication of Optical Fiber Mechanical Shock Sensors for the Los Alamos HERT (High Explosive Radio Telemetry) Project}

Federal Manufacturing \& Technologies

P. E. Klingsporn, D/MX4

\section{KCP-613-8038}

Distributed October 2005

Interim Report on HERT Project No. 70801675

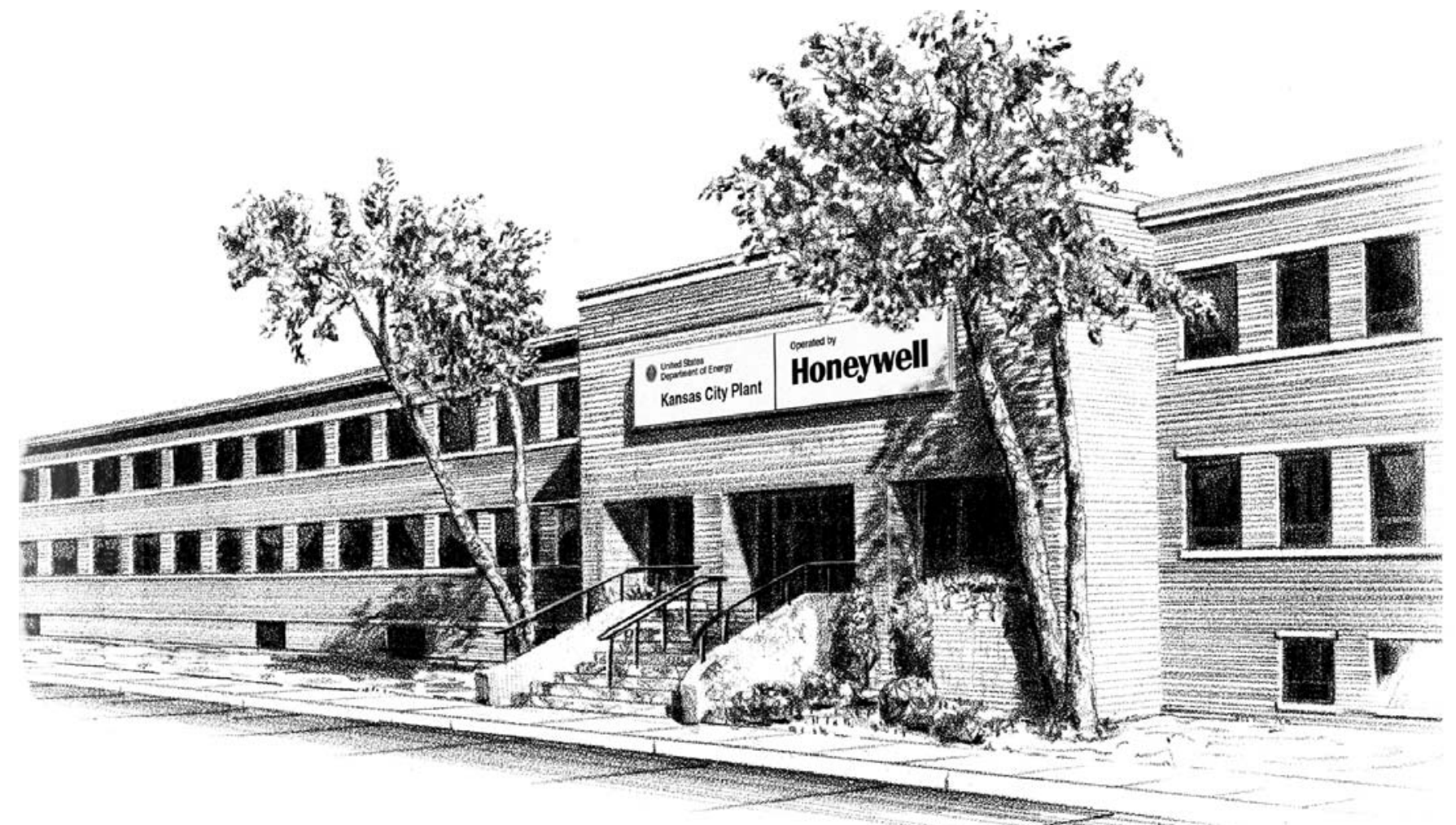

Prepared under prime contract DE-ACO4-01AL66850 for the

United States Department of Energy 


\section{DISCLAIMER}

This report was prepared as an account of work sponsored by an agency of the United States Government. Neither the United States Government nor any agency thereof, nor any of their employees, makes any warranty, express or implied, or assumes any legal liability or responsibility for the accuracy, completeness, or usefulness of any information, apparatus, product, or process disclosed, or represents that its use would not infringe privately owned rights. Reference herein to any specific commercial product, process or service by trade names, trademark, manufacturer, or otherwise, does not necessarily constitute or imply its endorsement, recommendation or favoring by the United States Government or any agency thereof. The views and opinions of authors expressed herein do not necessarily state or reflect those of the United States Government or any agency thereof.

All data prepared, analyzed and presented has been developed in a specific context of work and was prepared for internal evaluation and use pursuant to that work authorized under the reference contract. Reference herein to any specific commercial product, process or service by trade name, trademark, manufacturer, or otherwise, does not necessarily constitute or imply its endorsement, recommendation or favoring by the United States Government, any agency thereof or Honeywell Federal Manufacturing \& Technologies, LLC.

Printed in the United States of America.

This report has been reproduced from the best available copy.

Available to DOE and DOE contractors from the Office of Scientific and Technical Information, P.O. Box 62, Oak Ridge, Tennessee 37831; prices available from (865) 576-8401, FTS 626-8401.

Available to the public from the National Technical Information Service, U.S. Department of Commerce, 5285 Port Royal, Rd., Springfield, Virginia 22161, (703) 487-4650.

A prime contractor with the United States Department of Energy under Contract Number DE-AC04-O1AL66850

\author{
Honeywell Federal Manufacturing \& Technologies \\ P.O. Box 419159 \\ Kansas City, Missouri, 64141-6159
}




\section{Honeywell}

KCP-613-8038

Distribution Category UC-42

Approved for public release; distribution is unlimited.

Fabrication of Optical Fiber Mechanical Shock Sensors for the Los Alamos HERT (High Explosive Radio Telemetry) Project

P. E. Klingsporn, D/MX4

KCP-613-8038

Distributed October 2005 



\section{Contents}

Section Page

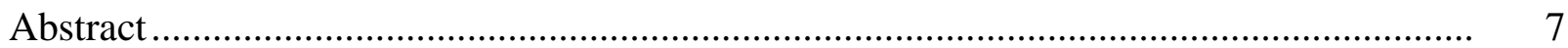

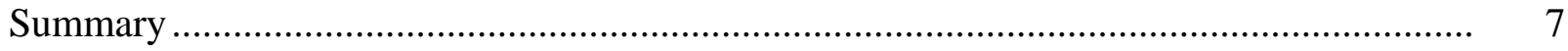

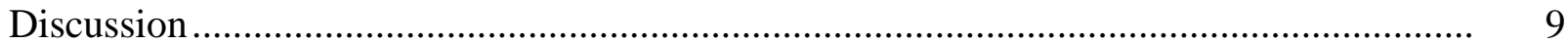

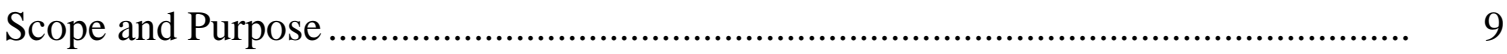

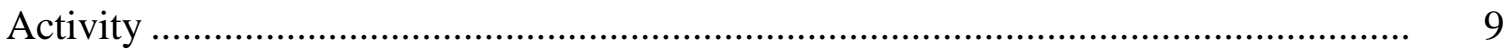

Requirements .................................................................................. 9

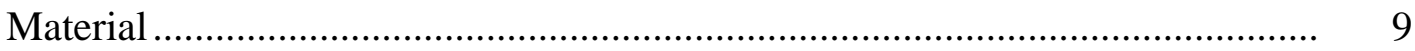

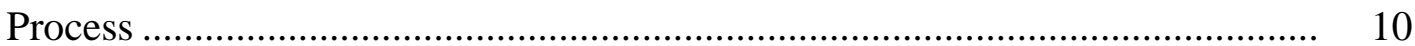

Testing and Acceptance ..................................................................... 15

Assembly of Fiber Sensors into a Sensor Cable .............................................. 17

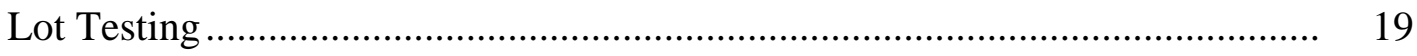

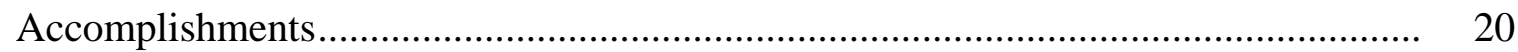




\begin{abstract}
This document lists the requirements for the fiber optic mechanical shock sensor for the Los Alamos HERT (High Explosive Radio Telemetry) project and provides detailed process steps for fabricating, testing, and assembling the fiber shock sensors for delivery to Los Alamos.
\end{abstract}

\title{
Summary
}

The requirements for the mechanical shock sensor for the Los Alamos HERT project are given in detail, along with fabrication, inspection, and assembly process steps. A key feature of the sensor is that the integrity of the glass optical fiber must be verified just prior to use; otherwise, if the fiber were broken, false optical signals could be registered. To assure the integrity, light is sent into the fiber, reflected from a coating on the sensor tip, and the return signal is used as an indicator that the fiber is not broken. To obtain maximum return signal, the tip must be polished flat, smooth, and the gold reflective coating must provide a minimum reflectance of 50 percent. The gold coating must be free of pin holes as measured by light percentage leakage of $10^{-6}$ or less. To assure quality coating, the polished fiber end must be cleaned adequately and the underlying chromium coating thickness must be controlled prior to application of the gold. After coating of gold, all fibers are inspected to determine that the internal reflectance at $850 \mathrm{~nm}$ wavelength is equal to or greater than 50 percent.

Since Lutetium Oxyorthosilicate: Cerium doped (LSO) produces light when subjected to mechanical shock, it is applied to the gold-coated fiber tip to provide enhanced light. The process for applying LSO involves dipping the fiber tip into a binder and then touching the tip to the surface of LSO powder, and then allowing the LSO-coated tip to dry for 24 hours. After drying, the LSO-coated tip is spray painted with flat black paint that serves to block the fiber tip from light generated in the HE detonation itself. After application of LSO and paint, the fiber sensors are again tested for internal reflectance to assure that the reflective coating was not damaged during LSO application.

First, a procedure for assembling acceptable fiber sensors into arrays of 8, 12, or 16, as the particular case may be, based on the sensor array requested by Los Alamos, is provided. Each fiber sensor is marked with a combination of black and red ink dots, near the tip, to provide fiber sensor identity when the sensors are later placed on the HE (High Explosive) at Los Alamos.

Finally, a procedure for securing the fragile optical fiber sensors to a heavy-duty cardboard base for packing in foam for subsequent shipment to Los Alamos, is provided. 


\section{Discussion}

\section{Scope and Purpose}

This document provides details for the materials, fabrication steps, assembly, and testing of optical fiber mechanical shock sensors for the Los Alamos HERT project. This document was compiled to provide a detailed description of the overall process for fabricating and testing the shock sensors prior to delivery to Los Alamos for their placement on HE (High Explosive).

\section{Activity}

\section{$\underline{\text { Requirements }}$}

Polished fiber end surface

- Smoothness $2.5-3.0 \mathrm{~nm}$ rms

- Flatness $1 \mu \mathrm{m}$

Chromium adherence coating thickness

- 25 - 30 Angstroms

Gold coating

- $\quad 2-2.5 \mu \mathrm{m}$ on polished fiber end

- $1-2.5 \mu \mathrm{m}$ on cylindrical fiber surface

- 4-6 mm length on cylindrical surface

- Fully dense (no pin holes)

Internal reflectance of gold coating*: $50 \%$ or greater at $850 \mathrm{~nm}$

Light Leakage*

- Inside fiber to external: $<10^{-6}$

- External of fiber to internal: $<10^{-8}$

* Applies both before and after LSO and blinder paint are applied.

\section{Material}

Multimode step-index optical fiber. Silica core, silica cladding, Polyimide buffer coating. Polymicro FVP 100/110/125 $\mu \mathrm{m}$. 


\section{$\underline{\text { Process }}$}

\section{Fiber Tip Polishing}

1. Cut a fiber segment approximately 2 inches longer than the final length required by LANL for the given application.

2. Mount one end of the fiber in Tool FP1 and allow 0.010 inch of fiber to extend beyond the end of the holder. Place Buehler No. 69-3121 paper on a flat granite slab and lap the fiber end until flush with the holder end. Clean the end and clean the tool to remove all grit.

3. Extend the fiber end 0.003 inch beyond the holder end and use Buehler No. 69-3172 paper to lap the fiber end flush with the holder end. Clean the fiber end and clean the tool to remove all grit.

4. Extend the fiber end $0.001-0.002$ inch beyond the holder end, and use Buehler No. 156801 film to polish the fiber end. Clean the fiber end and the holder.

5. Examine the fiber end with the Ultrastar Met Microscope. Measure flatness by using the microscope vertical stage vernier and focus alternately at fiber center and at fiber edge. If the difference exceeds $1 \mu \mathrm{m}$, repeat Step 4. In making these measurements, use a magnification of $200 \mathrm{X}$ and, if necessary to achieve resolution, use 500X.

6. Examine the fiber end surface smoothness, using the Ultrastar Met Microscope, using 200X (500X, if necessary). Assure that the surface is free of chips, cracks, pits, and scratches. The surface must have a uniform appearance. If isolated defects are visible, repeat Steps 3, 4, and 5.

7. Repeat Steps 1 through 6 for the desired number of fibers.

\section{Alternate Fiber Tip Polishing}

1. Cut sixteen fiber segments, each approximately 2 inches longer than the final length required by LANL for the given application.

2. Insert one end of each fiber into the holder of Tool FPMT 2, allowing the fiber ends to extend approximately 0.030 inch beyond the end of the MT. Melt a small quantity of Crystal Bond Wax around the ends of the fibers and inside the MT epoxy window to secure the fibers in place.

3. Lap with Buehler No. 69-3121 paper until the fiber ends are within approximately 0.005 inch of the end of the MT. Clean thoroughly. 
4. Lap with Buehler No. 69-3172 paper until the end of the MT is reached. Clean thoroughly.

5. Polish the fiber ends with Buehler No. 15-6801 film. Clean thoroughly.

6. Examine the fiber ends with the Ultrastar Met Microscope as in Steps 5 and 6 of Fiber Tip Polishing I. If any fibers do not meet the smoothness and flatness requirements, identify them for removal by cutting.

7. Use acetone to dissolve the wax, and carefully remove the fibers from the holder.

8. Repeat Steps 1 through 7 to obtain the desired number of fibers.

\section{Cleaning of Fiber Ends Just Prior to Gold Coating}

1. Mount the fiber ends in the cleaning fixture.

2. Clean ultrasonically for 20 seconds in DI water with the polished fiber ends down.

3. Rinse for 10 seconds in isopropyl alcohol with polished ends down.

4. Flush the fiber ends with alcohol for 20 seconds with the fibers vertical and the polished ends up.

5. Allow the fibers to dry in a clean bench in the absence of forced air.

\section{Coating of Gold on Polished Fiber Ends}

1. Install the chromium and gold sputtering sources in the head of the vacuum coating chamber.

2. Blow the fiber tips with nitrogen and load the fibers into the final holding device.

3. Adjust exposed tips of fibers to length of $5 \mathrm{~mm} \pm 1 \mathrm{~mm}$ and install the device on the platform in the vacuum coating chamber. Load a microscope slide so that it will be exposed to the sources in the same way as the fiber tips.

4. Close the coating chamber and evacuate to a level of $10^{-6} \mathrm{~mm} \mathrm{Hg}$. Bake the chamber walls to remove any moisture.

5. Back fill the chamber with Argon until a pressure of $10^{-3} \mathrm{~mm} \mathrm{Hg}$ is obtained.

6. Rotate the upper plate of the chamber so that the chromium source is exposed and energize the source. 
7. Rotate the lower platform containing the exposed fiber ends at a constant speed allowing it to pass once beneath the energized chromium source. NOTE: The $\mathrm{dC}$ magnetron sputtering voltage/current and the rotation rate of the fibers on the platform were determined in advance to provide 25-30 Angstroms of chromium on a microscope slide that had been placed in the same position relative to the source as the fibers later would occupy. This was done by allowing several passes of the plate beneath the chromium source at constant speed and then measuring the chromium thickness deposited on the slide. In this way, the thickness per one pass was calculated, with the result that approximately 25-30 Angstroms are deposited for each pass under the chromium source.

8. De-energize the chromium source. Without interrupting the coating chamber vacuum level (still at $10^{-3} \mathrm{~mm} \mathrm{Hg}$ from Step 5), rotate the upper chamber plate to cover the chromium source and expose the gold sputtering source. Energize the gold source with the $\mathrm{dC}$ magnetron sputtering voltage/current conditions that had been determined by previous tests to give a measured gold thickness on a microscope slide placed at the same distance from the gold source as the fiber tips now occupy.

9. Rotate the lower platform containing the microscope slide and fiber tips to be coated into the position beneath the source and allow deposition to proceed for 35.5 minutes, as determined in the pre-testing referred to in Step 8 that had resulted in a gold thickness of $2-2.5 \mu \mathrm{m}$ on the microscope slide.

10. De-energize the gold source. Allow the system and fibers to cool. Vent the chamber.

11. Taking care not to touch the coated fiber tips or to allow the tips to touch one another, remove the fibers from the chamber and carefully remove the fibers from the holding device. Remove the microscope slide from the chamber and hold it for subsequent gold thickness measurement.

\section{Application of LSO and Black Paint Blinder Coatings}

1. Place one or two drops of the SBE binder (CERAK M 700, Cetek Ceramic Technologies, Berea, $\mathrm{OH}$ ) in a small plastic boat. Incline the boat slightly so that the drop will move to a location with a minimum surface area. This should help to minimize the rate at which evaporation and/or the formation of a surface film occurs.

2. Place a very small quantity of the LSO crystals (ZBK58/F-S1:LuSiO5:Ce, Phosphor Technology, Essex EN9 2LP, England) in a separate plastic boat. Use a volume of only 0.01 to $0.02 \mathrm{~cm}^{3}$. This amount will occupy a cone-shape with a circular base of approximate diameter $1 / 4$ inch and height 0.04 to 0.08 inch.

3. Take the end of the first fiber to be coated and bring it just into contact with the surface of the SBE binder. In doing this, approach the SBE drop in a direction nearly perpendicular to the surface of the drop. (NOTE: After exposure of the SBE to air for 
20-30 minutes, it may develop a surface film or become too viscous. Tilting the plastic boat may allow less viscous binder to be exposed but, most likely, the drop may need to be discarded and fresh binder added to the boat.)

4. As quickly as possible after Step 3, bring the SBE-coated fiber end into contact with the surface of the LSO crystals (again taking care to approach the LSO surface with the fiber nearly perpendicular to it). Do not force the fiber end into the LSO, but merely bring it into contact with the surface. Forcing the fiber into the LSO crystals can possibly damage the reflective gold coating. Moreover, it causes too much of the LSO to be transferred to the fiber end.

5. View the fiber tip with the unaided eye. Often times the mass of crystals transferred in Step 4 is too great and this can easily be seen by the unaided eye. Excess LSO can be removed by holding the fiber firmly with one hand and then using the thumb and forefinger of the opposite hand to "thump" or otherwise impart momentum to the thumb of the hand holding the fiber, thereby dislodging the excess LSO. If this is not effective, try it again. To make a definitive judgment regarding the size of the LSO mass on the fiber tip, view it with a microscope and estimate the outer diameter of the LSO in relation to the fiber diameter.

6. The depth of the LSO crystals on the end of the fiber should be in the range .03 to .05 $\mathrm{mm}$. i.e., 30 to 50 percent of the fiber core diameter. For LSO crystals $8 \mu \mathrm{m}$ in diameter, this corresponds to approximately four to eight crystal diameters deep. The diameter of the LSO coating should not exceed two fiber diameters, or approximately 0.010 to 0.012 inch. From a functional standpoint, LSO is needed only on the end of the fiber, but it is acceptable if some LSO is on the cylindrical surface of the fiber, provided it does not extend more than 1 to $1.2 \mathrm{~mm}$ from the fiber end and provided the diameter is not more than 0.010 to 0.012 inch.

NOTE: If the LSO occupies too large a diameter and the "thumping" procedure described above is not successful in dislodging the excess LSO, repeat the process beginning with Step 3. To do this, dip the LSO/SBE-coated fiber end in the SBE (inserting it far enough to submerge the LSO entirely). After a few seconds, the LSO will come off and sink to the bottom of the SBE drop. Then, repeat the process, beginning with Step 3.

7. Place the fiber in a safe location to allow drying for a minimum of 24 hours.

8. Proceed to the next fiber and repeat Steps 1 through 6.

9. Secure the LSO-coated fiber ends for application of black paint. A manila folder will work well for this. Place a piece of double-sided tape at the end on the inside of one of the folder faces. Mount each fiber on the tape with its length perpendicular to the folder end edge but allow only 0.050 inch of the LSO-coated fiber end to extend beyond the folder end edge. Secure as many as 12 to 16 fibers in this way, allowing $1 / 8$ to $1 / 4$ inch separation between adjacent fibers. Then, close the other folder face down on the first 
face taking care to align the folder end edge flush with the folder end edge of the face on which the fibers are mounted. Gently press the second folder face into the tape that holds the fibers together on the first folder face. In addition, it is a good idea to secure the two folder ends with two pieces of tape placed at both ends of the array of exposed fiber ends but well away from the end fibers.

10. Next, follow the directions on the can of Krylon (No. 1602) ultra-flat black spray paint regarding preparation of the contents for application. Hold the manila folder in a vertical direction with the exposed fibers pointing upward. Hold the nozzle of the spray can at approximately 10 inches from the fiber ends. The directed line of spray from the nozzle to the fibers should be inclined at approximately $30^{\circ}$ above the horizontal plane through the fiber tips, and approximately $45^{\circ}$ relative to the folder face. Activate the spray and move the can from left to right, exposing the fibers for a period of one to two seconds. Repeat this operation, proceeding in the opposite direction from right to left. Repeat both passes with the spray directed at the opposite side of the folder. Place the folder in a vertical position with the exposed fiber tips up, and allow the paint to dry for a minimum of four hours.

11. Carefully remove the tape from the folder ends. Open the folder and carefully remove the fiber ends from the double-sided tape and place the fibers in a secure location until the next operation in the overall fiber-probe fabrication process.

Optional: After 10-15 minutes, repeat Step 10 if observation of the fiber ends appears to warrant it.

\section{Fiber Test Assembly Preparation}

1. Place 16 fibers, lying flat and nearly parallel to one another, on a horizontal surface with the gold coated ends all facing "to the right." The surface should be covered with a finetexture paper so that the fibers do not come in contact with metal or other hard surfaces or edges that can easily abrade the thin protective Polyimide buffer coating on the fiber.

2. Use a fine-tip black permanent marker ("Sharpie") to make one, two, three, and four small dots at the opposite ends ("on the left") of the first four fibers, respectively, to designate these fibers as Fibers 1, 2, 3, and 4. Place the dots within approximately 2 inches of the fiber ends.

3. Using a fine-tip red permanent marker (“Sharpie”), place one small dot within approximately 2 inches of the "left" ends of the next four fibers, and then place one, two, three, and four black dots next to the red dots to designate these as Fibers 5, 6, 7, and 8, respectively.

4. Using the red marker, place two small red dots within 2 inches from the "left" ends of the next four fibers, and then place one, two, three, and four black dots near each of the two red dots to designate these as Fibers 9, 10, 11, and 12, respectively. 
5. Using the red marker, place three small red dots within 2 inches of the "left ends" of the remaining four fibers, and then place one, two, three, and four black dots near each of the three red dots to designate these as Fibers 13, 14, 15, and 16, respectively.

6. Place a 16-hole MT ferrule on the table near the ends of the fibers with the black and red dots, and orient the MT so that the rectangular epoxy well is "up" and the insertion end of the ferrule is to the "right." Begin with Fiber No. 1, and insert it into the lowermost fiber position of the MT, when viewed from above. Allow approximately 0.050 inch of the fiber to extend through the end of the MT, to the left. Insert Fiber No. 2 into the adjacent fiber position in the MT. Continue inserting fibers in order until the last fiber, No. 16, is inserted into the uppermost fiber position of the MT.

7. Mix epoxy (Fiber-Fix FA-06) thoroughly in a small, clean aluminum cup that has a smooth bottom (63 ml, Fisher). Use a clean metal pick to transfer some epoxy to the top of the fiber ends that extend 0.050 inch beyond the end of the MT ferrule. Use the pick to transfer some epoxy to the lower portions of the fiber ends. With the pick positioned vertically, transfer a small amount of epoxy to the outer sides of Fibers 1 and 16, taking care not to get any epoxy into the alignment pin holes of the MT. Finally, transfer epoxy into the rectangular epoxy well of the MT, but take care not to fill above the level of the outer face of the MT.

8. Allow the epoxy to cure at room temperature for a minimum of 3 hours. Alternatively, place the MT with fibers in it into an oven and cure at $90^{\circ} \mathrm{C}$ for 20 minutes. NOTE: Elevated temperature curing of the epoxy is not necessary and it involves a certain level of handling risk in that only a short portion (4-6 inches) of the fibers should be in the oven. This means that the oven door should not be tightly closed on the fibers at their point of exit.

9. When the epoxy is cured, mount the MT in the Tool FPMT2. Place Buehler No. 69-3121 paper on a flat granite slab and lap the fiber ends to within approximately 0.005 inch of the end of the MT ferrule. Clean the end thoroughly and lap on Buehler No. 69-3172 paper until the end of the MT ferrule is reached. Clean thoroughly and polish the fiber ends on Buehler No. 15-6801 film.

10. Clean the polished ends thoroughly and examine each fiber with the Ultrastar Met Microscope for both flatness and surface smoothness, using the procedure in Steps 5 and 6 of Process I, Fiber Tip Polishing. If any fiber fails to meet the in-process inspection, repeat the steps with Buehler No. 69-3172 paper and Buehler No. 15-6801 film.

11. Repeat Steps 1 through 10 for the desired number of fibers. 


\section{Testing and Acceptance}

\section{Fiber with Gold Coating}

1. Connect the HP8153A to its power source and allow 20 minutes for equilibrium operational temperature to be reached. Connect the Newport 1830-C unit to its power source and allow 20 minutes for equilibrium. Follow the manual instructions for each unit to set the detector outputs at zero, in the absence of light, after the 20-minute period. Set the wavelength responsivity for $850 \mathrm{~nm}$ on both the HP8153A and the Newport 1830-C units. Connect the detector cable from the Newport integrating sphere to the input of the Newport 1830-C. Carefully group the gold coated ends of the 16 fibers together and insert them into the holder in the input port of the Newport integrating sphere (see Appendix A).

2. Connect the source end of Cable $1 \times 2$ SFC to the HP81541 source and connect the sensor end of Cable $1 \times 2$ SFC to the HP81530A sensor.

3. Connect the MT containing the 16 test fibers (obtained from Step 10 in Process V, Fiber Test Assembly Preparation) to the MT end of the $1 \times 16$ MT/ST cable, taking care that Fiber Number 1 of the MT test fiber array mates with Fiber Number 1 of the MT on the $1 \times 16 \mathrm{MT} / \mathrm{ST}$ cable.

4. Connect the No. 1 ST of the $1 \times 16 \mathrm{MT} / \mathrm{ST}$ cable to the ST end of $1 \times 2$ SFC cable. Record the sensor reading of the HP8153A. Record the Newport 1830-C reading as Internal Leakage. Continue, in turn, connecting each additional ST of the $1 \times 16$ MT/ST cable to the ST end of $1 \times 2$ SFC, and record the HP8153A reading and the Newport $1830-\mathrm{C}$ reading.

5. Disconnect the sensor and source ends of cable $1 \times 2$ SFC from the HP8153A unit. Connect the sensor end of ST/ST J cable to the 81530A sensor of the HP8153A.

6. Carefully remove the gold-plated fiber ends from the input holder of the port on the Newport integrating sphere. Insert gold-coated Fiber No. 1 end in the input port of Fixture Ext. L, and connect the Number 1 fiber of the $1 \times 16 \mathrm{MT} / \mathrm{ST}$ cable to the output ST connector of the ST/ST J cable. Connect the source of Ext. L to power. Record the HP8153A sensor reading as External Leakage. Remove goldcoated Fiber 1 end from Ext. L, and insert gold-coated Fiber Number 2 end. Connect Number 2 fiber of the $1 \times 16 \mathrm{MT} / \mathrm{ST}$ cable to the output ST of the ST/ST J cable, and record the HP8153A sensor reading. Continue in this way until all 16 gold-coated fiber ends have been measured. 
7. Identify those fibers (if any) for which any one of the following is true:

- $\quad$ HP reading in Step 4 is $<2.14 \mu \mathrm{W}$.

- Newport reading in Step 4 is $>0.02 \mathrm{nW}$.

- $\quad$ HP reading in Step 6 is $>1.7 \mathrm{pW}$.

8. A fiber for which any one of the conditions (a), (b), or (c) is true does not meet the requirements for coating reflectance, internal-to-external leakage, and external-to-internal leakage. Note and record in the BUILD BOOK any unusual circumstances concerning any of the testing.

9. Cleave all 16 fibers as near the rear of the MT ferrule as possible. Remove any fiber(s) from the group that were identified in Step 7.

10. Measure the gold coating length. Reject any fiber if the coating is not in the range 4-6 mm. Inspect the coatings and note any unusual appearance in the BUILD BOOK.

11. Proceed with the next group of 16 fibers for testing.

NOTE: Work is underway to assemble a "golden" fiber sensor test cable that can be used on a frequent basis to monitor the readings obtained for reflectance in 7(a), internal-to-external leakage in 7(b), and external-to-internal leakage in 7(c). The intent is to have three fibers for each of the tests, 7(a), 7(b), and 7(c). In each group of three, one fiber would be a failure, one would be nominal, and one would exceed the required reading significantly. Prior to testing a group of fibers, the "golden" fiber cable will be tested and, if the readings are in range, the testing can proceed. If the "golden" test fibers do not respond properly, then diagnostics will be required. This will involve inspection of cable ends for damage on Cable $1 \times 2$ SFC, $1 \times 16$ MT/ST cable, and the ST/ST J cable, as well as measurement of the HP81541 source and the lamp source strength on Fixture Ext. L. In addition, periodic calibration of the HP8153A and Newport systems will be conducted through the Metrology Department.

\section{Fibers with LSO and Blinder Coatings on Gold}

Follow Steps 1 through 10 in Section I for testing and acceptance of fibers with gold coating. 


\section{Assembly of Fiber Sensors into a Sensor Cable}

1. Select 16 acceptable fibers that passed Step 7 after LSO coating, and place them on a flat surface, nearly parallel, with the LSO-coated ends oriented "to the right." Disregard the red and black dots at the left ends of the fibers that had served to identify the fibers for reflectance and leakage testing.

2. Use a piece of Kapton tape to carefully secure the fiber to the table surface within approximately 3 inches from the LSO-coated end. Place the left end of the fiber under axial tension (one to two pounds) so that the fiber is straight and cleave the left end at the length prescribed by Los Alamos for the particular application. Place one black dot on the fiber near the LSO-coated end to designate the fiber as Fiber Sensor 1.

3. Continue the procedure outlined in Step 2 to cut each fiber to the prescribed length, and place the appropriate number of black and/or red dots on the fibers to designate their identifying numbers. The tabulation below gives the number of black/red dots to be used to designate the fiber sensor number.

\begin{tabular}{|c|c|c|}
\hline Fiber Number & Black/Red Dots & Fiber Length* $^{*}$ \\
\hline 1 & B & \\
\hline 2 & BB & \\
\hline 3 & BBB & \\
\hline 4 & BBBB & \\
\hline 5 & R & \\
\hline 6 & RB & \\
\hline 7 & RBB & \\
\hline 8 & RBBB & \\
\hline 9 & RBBBB & \\
\hline 10 & RR & \\
\hline 11 & RRB & \\
\hline 12 & RRBB & \\
\hline 13 & RRBBB & \\
\hline 14 & RRBBBB & \\
\hline 15 & RRR & \\
\hline 16 & RRRB & \\
\hline
\end{tabular}

* Prescribed by LANL for particular application.

4. Cut a piece of Teflon (or shrink) tubing approximately 1 inch long and insert it through the oval-shaped hole in the stainless steel fitting, SSF No. 1, and then position it so that nearly equal lengths extend past either end of SSF No. 1. Place the fitting just beyond the left ends of the 16 fibers and orient it so that the oval-shaped end is nearest the ends of the fibers.

5. Place a 16-hole MT to the left of the SSF No. 1 fitting with its epoxy well up and its fiber-insertion end nearest the rectangular cavity end of the SSF NO. 1. Take the left end of Fiber Sensor No. 1, and feed it through the protective tube in SSF No. 1, and then 
insert it into the lowermost fiber position in the 16-hole MT. Allow the fiber end to extend past the MT end (later to be polished) by a distance of 0.050 inch. Continue with Fiber Sensor No. 2, passing its left end through the tubing and into the adjacent fiber position relative to Fiber No. 1 in the MT. Continue until Fiber No. 16 is passed through the tubing and placed into the uppermost fiber position, No. 16 in the MT. Make sure all the fiber ends extend through 0.050 inch beyond the flat end of the MT.

6. Mix epoxy (Fiber-Fix FA-06) thoroughly and apply it to the 16 fibers in the MT per the process given in Steps 7 and 8 under Process for Fiber Test Assembly Preparation. Allow the epoxy to cure.

7. Follow Steps 9 and 10 of Process for Fiber Test Assembly Preparation to polish and inspect surface smoothness and flatness of the 16 fibers assembled into the $1 \times 16 \mathrm{MT}$ ferrule.

8. Mix thoroughly a small quantity (6 grams) of 3M Scotch-Weld $2216 \mathrm{~B} / \mathrm{A}$ epoxy in a smooth-bottom aluminum cup and use a metal pick to apply a small amount at the top and down the outside a distance 0.030 inch (all the way around) at the insertion end of the MT ferrule. Retract the tube inside the SSF No. 1 so that its end is flush with the bottom surface of the rectangular cavity. Carefully slide the SSF No. 1 toward the end of the MT that has been prepared with epoxy and force the rectangular cavity firmly over the fiberinsertion end of the MT ferrule. Allow the epoxy to cure at room temperature for 24 hours.

9. Cut the proper length of Teflon protective jacket required by LANL for the particular application, and carefully slip it over the LSO-coated fiber sensor ends and up to and in contact with the rear of the oval-shaped end of the SSF No. 1 fitting. Cut a 2 inch long piece of shrink tubing that is just large enough to slide over the oval-shaped end. Slip this over the LSO-coated ends and over and along the Teflon tubing and over the ovalshaped end of SSF No. 1 until it is in contact with the flange on the SSF No. 1 fitting. Use a hot air gun to heat and shrink the tubing down over the oval-shaped end and along the Teflon tubing, thus securing the Teflon tubing to the end of the SSF No. 1 fitting.

10. Carefully bend the $1 \times 16$ fiber sensor cable into a circle approximately 12 inches in diameter, and secure with Kapton tape to a $1 / 4$ inch thick fiberboard that is 18 inches square. Use Kapton tape to secure the connector end to the board, but do not allow the tape to contact the polished fiber ends. Slip a 4 inch long piece of Teflon tubing over the LSO-coated ends of the fibers, and tape the tubing to the board. Place a piece of Kapton tape over the end of the Teflon jacket, and secure it to the board. Place the board in a box with foam below and above, and ship to LANL. See Appendix B for an example of the HERT 11 and 12 cable specification. (Note: For HERT 11 and 12, two 8-hole MTs are used in place of a single 16-hole MT. However, the fiber numbers and designated black/red dots are used sequentially so that the fibers in the two 8-hole MTs are combined into a single 16-hole MT that is connected to a 16-hole MT port on the HERT electronics housing.) 


\section{Lot Testing}

\section{Non-Destructive}

1. Measure the gold coating thickness on the microscope slide that was in the vacuum coating chamber at the same time that a given group of fiber ends were coated.

2. Use the WYKO interferometer to measure the rms surface smoothness of one in sixteen polished fiber ends. This fiber can then be included with a group of fibers for gold coating.

\section{Destructive}

1. Select one in sixteen fiber sensors for sectioning and measurement of:

- Gold coating thickness on polished fiber end

- Gold coating thickness on the cylindrical "side" of the fiber

- LSO coating thickness

- Blinder coating thickness

2. Enter results in BUILD BOOK. 


\section{Accomplishments}

To avoid the possibility of false data signals from optical fiber shock sensors, it is imperative to have a means of testing the fiber integrity, that is, whether the glass fibers are intact or broken prior to use. Three key features are necessary to provide a valid test. First, the fiber end must be polished sufficiently smooth so adequate levels of light will be reflected internally from a coating applied to the polished surface. Secondly, the polished end must be sufficiently flat so that light reflected from it will return at angles within the fiber numerical aperture and thereby propagate back to the detectors. Finally, the reflective coating applied to the polished fiber end must be of high quality to assure the maximum level of reflectance in the fiber at the wavelength used to test the fiber integrity.

Techniques were developed to achieve the necessary smoothness (50 Angstroms rms) and flatness (1 micrometer) of polish on the small diameter (100 micrometers) fiber core. A sputtering process was developed to achieve the maximum reflectance at $850 \mathrm{~nm}$, with a gold coating. In order for gold to adhere to the glass fiber end, a layer of either chromium or titanium must be applied first, followed by gold. However, if the adherence layer of chromium is too thick, then too much light will be absorbed before it reaches the gold, where the intended internal reflection occurs. On the other hand, if the chromium layer is too thin, the gold adherence will not be adequate. The chromium layer must be thick enough to provide gold adherence, but thin enough to allow adequate light to pass through it, be reflected from the gold, and then pass again through the chromium and into the fiber where it is transmitted at a high enough level to register at the detector used to indicate fiber integrity. A sputtering process was developed to achieve the proper chromium thickness (25 Angstroms) followed by 2 micrometers of gold that gave adequate retro-reflection in the fiber at the test wavelength of $850 \mathrm{~nm}$.

Arrival of the high explosive detonation shock wave at a given fiber sensor site results in mechanical shock-induced light in the fiber that is then transmitted to the corresponding detector in the HERT electronics package. Earlier work at Special Technologies Laboratories (STL), Santa Barbara, had shown that Lutetium Oxyorthosilicate, Cerium doped (LSO) generates light when it is subjected to mechanical shock. Tests at LANL using fiber sensors fabricated at the Kansas City Plant showed that shock-generated light enhancement occurred when a small quantity of LSO was applied to the fiber sensor tip. Because of this, the process for application of the LSO to the optical fiber sensor tips was transferred from STL to Honeywell FM\&T. A complete documentation of this process was developed at FM\&T and is included. 
Appendix A:

Test for Fiber Self-Check Reflectance and Light Leakage 


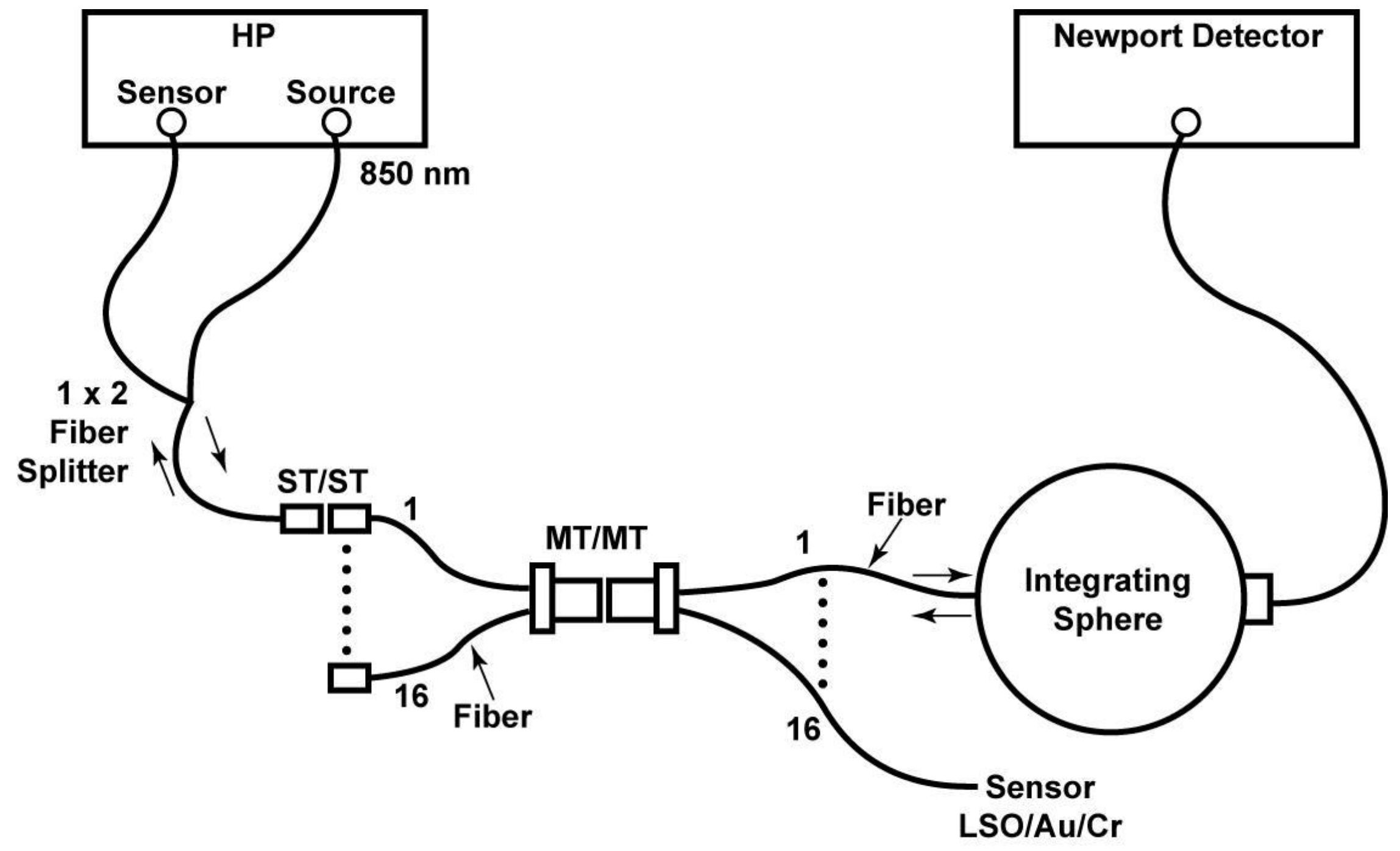

Figure A1: Test for Fiber Self-Check Reflectance and Light Leakage 


\section{Appendix B:}

\section{HERT 11 and 12 Fibre Optic}

Bundle Requirements 


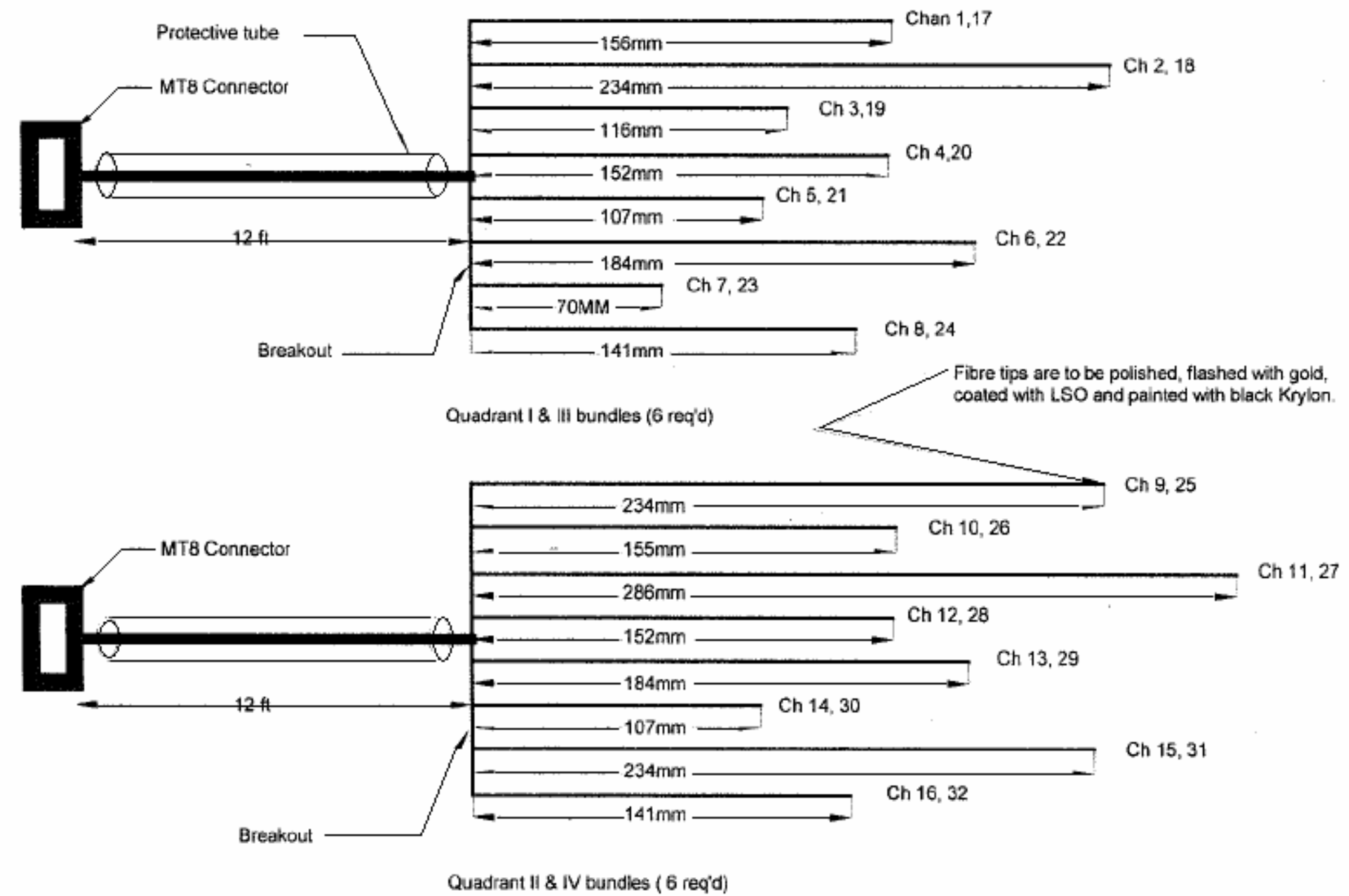

Figure B1: HERT 11 and 12 Fibre Optic Bundle Requirements 